\title{
DÉTERMINATION PAR DES MÉTHODES OPTIQUES DES TROIS COMPOSANTES D'UN CHAMP MAGNÉTIQUE TRÈS FAIBLE
}

\section{J. DUPONT-ROC}

Faculté des Sciences de Paris, Laboratoire de Spectroscopie Hertzienne de l'E. N. S., associé au C. N. R. S., 24, rue Lhomond, Paris $5^{\mathrm{e}}$

(Reçu le 7 août 1970)

\begin{abstract}
Résumé. - En raison de leur finesse, les résonances de croisement de niveaux observables en champ nul dans l'état fondamental des alcalins sont intéressantes pour la détection des champs magnétiques très faibles. En particulier, des champs inférieurs à 10-9 G ont été mesurés en ajoutant un champ de radiofréquence parallèlement au champ à mesurer. Nous montrons dans cet article que des résonances intéressantes apparaissent également lorsqu'on balaie le champ statique dans une direction quelconque par rapport au champ de radiofréquence ou lorsqu'on ajoute un deuxième champ de R. F. perpendiculaire au premier. Nous avons calculé rigoureusement la forme des signaux et nous les avons mis en évidence expérimentalement. Nous montrons qu'on peut utiliser ces signaux pour mesurer les trois composantes du champ magnétique : nous proposons pour cela deux méthodes de caractéristiques complémentaires, l'une utilisant un seul champ de R. F., la seconde en utilisant deux.
\end{abstract}

\begin{abstract}
The very narrow zero-field level-crossing resonances which can be observed in the ground state of optically pumped alkali atoms are of great interest for the detection of very weak magnetic fields. Fields as small as $10^{-9} \mathrm{G}$ have been measured by adding a R. F. field in the direction of the field to be measured. We show in this paper that resonances of interest also appear if the static field lies in an arbitrary direction with respect to the R. F. field, or if a second R. F. field is introduced perpendicularly to the first one. An exact calculation of the corresponding signals is given and their experimental observation is reported. We show that these signals can be used to measure the three components of the magnetic field; we propose two different methods with complementary characteristics, involving one and two R. F. fields respectively.
\end{abstract}

Introduction. - Des résonances de croisement de niveaux en champ nul, extrêmement fines (largeur de l'ordre de $1 \mu \mathrm{G}$ ) ont été observées récemment dans l'état fondamental d'alcalins pompés optiquement et ont été utilisées pour la détection et la mesure de champs magnétiques très faibles de direction donnée $[1,2,3]$.

Nous nous affranchissons dans le présent article de cette restriction sur la direction du champ et présentons les résultats d'une étude théorique et expérimentale de résonances qui apparaissent dans un champ de direction quelconque. Nous décrivons également deux méthodes n'utilisant qu'une cellule de résonance et un seul faisceau de pompage permettant de compenser un champ de direction quelconque, et par suite d'en mesurer les 3 composantes, avec une précision de l'ordre de $10^{-7}$ à $10^{-9} \mathrm{G}$.

Rappelons brièvement les conditions dans lesquelles apparaissent les résonances en champ nul décrites dans les références $[1,2]$. Les atomes contenus dans la cellule $C$ sont pompés optiquement dans l'état fondamental par un faisceau $F$ polarisé circulairement se propageant dans la direction Ox (Fig. 1). Ils sont soumis à l'action d'un champ de radiofréquence $\mathbf{H}_{1} \cos \omega t$ parallèle à $\mathrm{Oz}$. $\omega$ est grand devant l'inverse

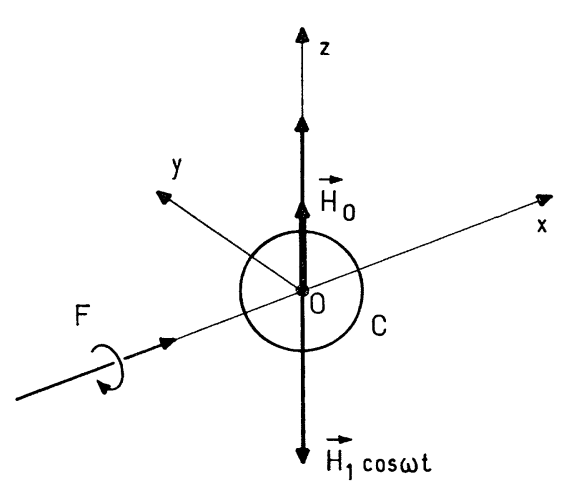

Fig. 1. - Disposition des champs et du faisceau de pompage dans une expérience de résonance paramétrique "ordinaire».

des temps de relaxation. On observe sur la lumière transmise par la cellule des modulations aux fréquences $p \omega / 2 \pi(p=0,1,2, \ldots)$, dont les amplitudes subissent des variations résonnantes lorsqu'on balaie le champ magnétique statique $\mathbf{H}_{0}$, parallèle à $\mathrm{Oz}$, autour de zéro $(*)$. Ces variations ont des formes de courbe de

(*) D’autres résonances apparaissent pour

$$
\gamma H=n \omega(n= \pm 1, \pm 2, \ldots)
$$

$\gamma$, rapport gyromagnétique. 
Lorentz en absorption pour les modulations paires, en dispersion pour les modulations impaires. L'étude complète de ces résonances, dites "résonances paramétriques ", a été publiée dans les références [4, 5].

Les modulations impaires (par exemple $p=1$ ) sont particulièrement intéressantes : au voisinage du champ nul (c.-à-d. dans un champ inférieur à la largeur de raie), l'amplitude de ces modulations est proportionnelle au champ $H_{0}$. Les variations de ce signal permettent de mesurer des variations extrêmement faibles du champ magnétique (de l'ordre de $10^{-9}$ à $10^{-10} \mathrm{G}$ ). Le magnétomètre ainsi réalisé a toutefois l'inconvénient de nécessiter la connaissance préalable de la direction du champ à mesurer afin de pouvoir orienter convenablement le montage expérimental. Or souvent dans la pratique, la direction du champ à mesurer est inconnue : il est donc nécessaire de connaître la réponse du magnétomètre à un tel champ. L'objet de la première partie sera donc l'étude des «résonances paramétriques » dans un champ $\mathbf{H}$ de direction quelconque. La forme des signaux est discutée en détail et des vérifications expérimentales effectuées sur ${ }^{87} \mathrm{Rb}$ sont présentées. Nous montrons comment on peut utiliser les signaux précédents pour mesurer et compenser les 3 composantes d'un champ magnétique à $10^{-9} \mathrm{G}$ près.

Dans une seconde partie, nous décrivons une autre méthode permettant d'atteindre le même but : en utilisant deux champs de radiofréquence, on peut obtenir sur la lumière transmise trois signaux indépendants, proportionnels respectivement aux trois composantes du champ magnétique régnant au niveau de la cellule et permettant de les mesurer simultanément. Nous analysons donc en détail les signaux de résonance apparaissant au voisinage du champ nul dans une expérience de pompage optique transversal en présence de deux champs de radiofréquence orthogonaux. Nous présentons également une étude expérimentale de la sensibilité de ce dispositif, réalisée sur ${ }^{87} \mathrm{Rb}$. Les avantages et les inconvénients des deux méthodes proposées dans cet article sont discutés en conclusion.

Dans un but de simplicité, nous ne donnerons ici que le principe général des calculs permettant d'établir l'expression des signaux observés. Le détail en est exposé en appendice ou ailleurs [6]. Nous nous attacherons par contre à discuter la forme des résultats, l'influence des divers paramètres et la façon dont ces diverses résonances peuvent être utilisées dans la pratique.

I. Résonance paramétrique dans un champ de direction quelconque. - 1 . CONDITIONS EXPÉRIMENTALES. - Les atomes, de moment cinétique $S$, pompés optiquement par le faisceau $F$ (parallèle à $\mathrm{Ox}$ ), sont soumis à l'action du champ $\mathbf{H}_{1} \cos \omega t$ (parallèle à $\mathrm{Oz}$ ) et du champ statique $\mathbf{H}$, de composantes $H_{\mathrm{x}}, H_{\mathrm{y}}$ $H_{z}$ (Fig. 2). Nous nous limitons à l'étude de la résonance en champ nul. Rappelons que nous supposons $\omega$ grand devant la largeur de cette résonance.

Pour simplifier le calcul, nous considérons un niveau qui n'a aucune structure hyperfine. Les résultats peuvent néanmoins être généralisés au cas de l'état fondamental des alcalins si on néglige les transferts d'orientation d'un niveau hyperfin à l'autre.

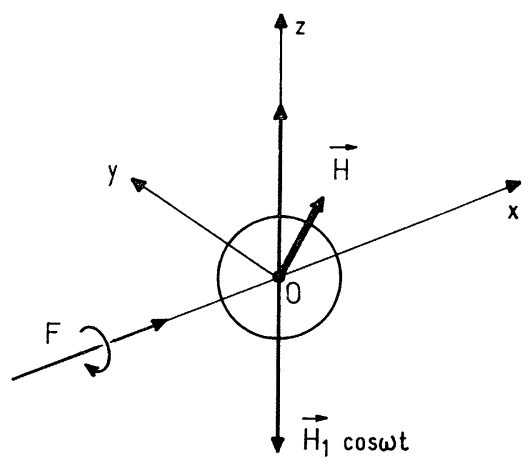

FIG. 2. - Résonance paramétrique dans un champ de direction quelconque.

2. Calcul des signaux. - Les équations d'évolution des composantes de l'orientation $\mathbf{M}=\langle\mathbf{S}\rangle$ s'écrivent :

$$
\left\{\begin{aligned}
\frac{\mathrm{d}}{\mathrm{d} t} M_{+}+\left(-i \omega_{\mathrm{z}}-i \omega_{1} \cos \omega \mathrm{t}\right) M_{+} & = \\
& =-2 i)_{+} M_{\mathrm{z}}+\lambda \\
\frac{\mathrm{d}}{\mathrm{d} t} M_{\mathrm{z}}+\Gamma M_{\mathrm{z}}=-i\left(\omega_{-} M_{+}-\right. & \left.M_{-} \omega_{+}\right)
\end{aligned}\right.
$$

où

$$
\begin{aligned}
M_{ \pm} & =\left\langle S_{\mathrm{x}} \pm i S_{\mathrm{y}}\right\rangle \\
\omega_{ \pm} & =\frac{\omega_{\mathrm{x}} \pm i \omega_{\mathrm{y}}}{2} \\
\omega_{\mathrm{x}} & =-\gamma H_{\mathrm{x}}, \ldots \\
\omega_{1} & =-\gamma H_{1}
\end{aligned}
$$

$\lambda$, terme constant réel, décrit l'introduction continue d'orientation dans la direction Ox par le pompage optique. $1 / \Gamma$ est le temps de relaxation de l'état fondamental sous l'action du pompage optique et des collisions contre la paroi (en toute rigueur, il faudrait introduire plusieurs constantes de temps différentes). $\gamma$ est le rapport gyromagnétique du niveau considéré.

Les deux équations (1) ont été écrites en regroupant aux premiers membres les termes d'évolutions propres de $M_{+}$et $M_{z}$ et, aux seconds membres, les termes linéaires en $\omega_{\mathrm{x}}$ et $\omega_{\mathrm{y}}$ qui couplent $M_{+}$et $M_{\mathrm{z}}$. Dans une expérience de «résonance paramétrique ordinaire », le champ magnétique est parallèle à $\mathrm{Oz}$, donc

$$
\omega_{\mathrm{x}}=\omega_{\mathrm{y}}=0 \text {. }
$$

Les deux équations sont alors découplées et leur solution s'obtient facilement. Soit $M_{+}^{(0)}$ et $M_{z}^{(0)}$ les solutions correspondantes. Lorsque $\omega_{x}, \omega_{y} \neq 0$, on peut chercher $M_{+}$et $M_{z}$ sous forme d'un développement en série entière en $\omega_{\mathrm{x}}$ et $\omega_{\mathrm{y}}$ [7]. 


$$
\begin{aligned}
& M_{+}=M_{+}^{(0)}+\cdots+M_{+}^{(n)}+\cdots \\
& M_{z}=M_{z}^{(0)}+\cdots+M_{z}^{(n)}+\cdots
\end{aligned}
$$

où $M_{+}^{(n)}$ et $M_{\mathbf{z}}^{(n)}$ sont d'ordre $n$ en $\omega_{\mathbf{x}}$ et $\omega_{\mathbf{y}}$.

Les équations (1) permettent de calculer $M_{+}^{(n)}$ et $M_{\mathrm{z}}^{(n)}$ en fonction de $M_{+}^{(n-1)}$ et $M_{\mathrm{z}}^{(n-1)}$, et de proche en proche en fonction de $M_{+}^{(0)}$ et $M_{z}^{(0)}$. Les termes d'ordre 2 sont par exemple calculés par cette méthode dans la référence [2].

Or, nous montrons en appendice que, non seulement on peut calculer explicitement tous les termes du développement, mais qu'on peut aussi resommer exactement la série de perturbation. On obtient ainsi la valeur exacte de l'orientation en régime stationnaire, quelle que soit la valeur de $|\gamma H|$ par rapport à la largeur $\Gamma$. La seule hypothèse nécessaire est $|\gamma H| \ll \omega$. Nous supposons donc que la résonance en champ nul à laquelle nous nous intéressons est nettement séparée de celles qui apparaissent dans les champs $\gamma H=n \omega$ $(n= \pm 1, \pm 2, \ldots)$.

On trouve ainsi :

$$
M_{\mathrm{x}}+i M_{\mathrm{y}}=(A+i B) \exp \left(i \frac{\omega_{1}}{\omega} \sin \omega t\right)
$$

où

$$
\begin{aligned}
A & =\frac{\lambda J_{0}}{\Gamma} \frac{\Gamma^{2}+\bar{\omega}_{\mathrm{x}}^{2}}{\Gamma^{2}+\bar{\omega}_{\mathrm{x}}^{2}+\bar{\omega}_{\mathrm{y}}^{2}+\bar{\omega}_{\mathrm{z}}^{2}} \\
B & =\frac{\lambda J_{0}}{\Gamma} \frac{\Gamma \bar{\omega}_{\mathrm{z}}+\bar{\omega}_{\mathrm{x}} \bar{\omega}_{\mathrm{y}}}{\Gamma^{2}+\bar{\omega}_{\mathrm{x}}^{2}+\bar{\omega}_{\mathrm{y}}^{2}+\bar{\omega}_{\mathrm{z}}^{2}} \\
M_{\mathrm{z}} & =\frac{\lambda J_{0}}{\Gamma} \frac{-\Gamma \bar{\omega}_{\mathrm{y}}+\bar{\omega}_{\mathrm{x}} \bar{\omega}_{\mathrm{z}}}{\Gamma^{2}+\bar{\omega}_{\mathrm{x}}^{2}+\bar{\omega}_{\mathrm{y}}^{2}+\bar{\omega}_{\mathrm{z}}^{2}} .
\end{aligned}
$$

On a posé :

$$
\bar{\omega}_{\mathrm{x}}=\omega_{\mathrm{x}} J_{0} ; \bar{\omega}_{\mathrm{y}}=\omega_{\mathrm{y}} J_{0} ; \bar{\omega}_{\mathrm{z}}=\omega_{\mathrm{z}} .
$$

Pour alléger les notations, on a écrit $J_{0}$ pour $J_{0}\left(\omega_{1} / \omega\right)$, valeur de la fonction de Bessel d'ordre 0 pour l'argument $\omega_{1} / \omega$. On appellera dans la suite $\boldsymbol{\Delta}$ le dénominateur

$$
\bar{\Delta}=\Gamma^{2}+\bar{\omega}_{\mathrm{x}}^{2}+\bar{\omega}_{\mathrm{y}}^{2}+\bar{\omega}_{\mathrm{z}}^{2}
$$

Les variations de la lumière absorbée par la cellule sont proportionnelles à $M_{\mathrm{x}}$ dont l'expression est donnée par

$$
M_{\mathrm{x}}=\operatorname{Re}\left\{(A+i B) \exp \left(\frac{i \omega_{1}}{\omega} \sin \omega t\right)\right\} .
$$

Soit encore :

$$
\begin{aligned}
M_{\mathrm{x}}=J_{0} A & +\sum_{q \geqslant 1}\left(2 J_{2 q} \cos 2 q \omega t\right) A \\
& -\sum_{q \geqslant 0}\left(2 J_{2 q+1} \sin (2 q+1) \omega t\right) B
\end{aligned}
$$

$J_{n}$ désigne la fonction de Bessel d'ordre $n$, d'argument $\omega_{1} / \omega$.

REVUE DE PHYSIQUe APPLIQUÉE. - T. 5, No 6, DÉCEMBRE 1970
3. DisCuSSION DE LA FORME GÉNÉRALE DES SIGNAUX. - On constate que l'amplitude de ces signaux est proportionnelle à l'intensité du pompage optique, caractérisée par $\lambda$; en fait $\lambda$ figure multiplié par le facteur $J_{0}$ qui est inférieur à 1 . On peut dire, en quelque sorte, que l'efficacité du pompage transversal est diminuée dans un rapport $J_{0}$ par la présence du champ de R.F. Ce phénomène a été étudié théoriquement et expérimentalement en détail dans les références $[8,9]$.

- La lumière transmise par la cellule est modulée à toutes les fréquences multiples de $\omega / 2 \pi$. Le signal modulé à un harmonique pair $2 q$ s'écrit

$$
\left(2 J_{2 q} \cos 2 q \omega t\right) A \text {, }
$$

celui modulé à un harmonique impair $2 q+1$ s'écrit $-\left(2 J_{2 q+1} \sin (2 q+1) \omega t\right) B$.

- L'existence de ces divers harmoniques $n \omega / 2 \pi$ et de leurs facteurs de pondération $J_{n}\left(\omega_{1} / \omega\right)$ provient de la modulation de la fréquence de Larmor introduite par le champ $\mathbf{H}_{1} \cos \omega t$.

- Les variations des signaux en fonction du champ statique sont données par les expressions $\boldsymbol{A}$ et $\boldsymbol{B}$. On voit clairement sur (4) et (5) que la variation d'une modulation quelconque en fonction d'une composante arbitraire du champ magnétique est de forme lorentzienne, en général un mélange des formes d'absorption et de dispersion.

- $\omega_{\mathrm{x}}$ et $\omega_{\mathrm{y}}$ ne figurent dans les expressions de $A$ et de $B$ que multipliés par le facteur $J_{0}$. Ce fait a une portée très générale. Le mouvement du moment magnétique d'un atome soumis simultanément à l'action d'un champ oscillant $\mathbf{H}_{1} \cos \omega t$ et d'un champ statique $\mathbf{H}$ faible (c.-à-d. tel que $|\gamma H| \ll \omega$ ) peut en effet être décomposé en deux composantes : un mouvement « lent » à des fréquences de l'ordre de $\gamma H / 2 \pi$ auquel se superposent les oscillations forcées « rapides » aux fréquences $p(\omega / 2 \pi)$. Le mouvement lent correspond à l'évolution moyenne du moment magnétique, la moyenne étant prise sur quelques périodes du mouvement rapide. Or, on a pu montrer [8, 9] que la fréquence du mouvement lent est différente de la fréquence de Larmor de l'atome dans le champ $\boldsymbol{H}$. La présence du champ oscillant modifie la fréquence de précession de l'atome dans des champs faibles. Cette modification dépend de l'orientation relative du champ statique et du champ de radiofréquence : si ces 2 champs sont parallèles, la fréquence de Larmor est inchangée; si au contraire, ils sont perpendiculaires la fréquence de Larmor est multipliée par $J_{0}\left(\omega_{1} / \omega\right)$.

Ainsi dans les formules (4) et (5), ce ne sont pas les fréquences de Larmor $\omega_{\mathrm{x}}, \omega_{\mathrm{y}}, \omega_{\mathrm{z}}$ de l'atome libre qui apparaissent, mais les fréquences $\bar{\omega}_{\mathrm{x}}, \bar{\omega}_{\mathrm{y}}, \bar{\omega}_{\mathrm{z}}$ de l'atome en présence du champ alternatif, appelé aussi dans les références [8,9] fréquences de Larmor de l' « atome habillé » par le champ R. F.

La présence des facteurs $J_{0}$ se traduira par un élar- 
gissement des courbes de résonance par un facteur $1 / J_{0}$ lorsqu'on balaye $H_{\mathrm{x}}$ ou $H_{\mathrm{y}}$. On voit en particulier que la largeur devient infinie pour les valeurs de $\omega_{1} / \omega$ qui annulent $J_{0}[8]$.

- La forme du dénominateur $\bar{\Delta}$ fait apparaître un élargissement de la courbe de résonance, si on balaye l'une des 3 composantes, alors que l'une des deux autres n'est pas nulle. Par exemple, si on balaye le champ $H_{\mathrm{y}}$, la largeur des courbes de résonance correspond à $\Delta \bar{\omega}_{\mathrm{y}}=2 \sqrt{\Gamma^{2}+\bar{\omega}_{\mathrm{x}}^{2}+\bar{\omega}_{\mathrm{z}}^{2}}$, soit

$$
\Delta \omega_{\mathrm{y}}=\frac{2}{J_{0}} \sqrt{\Gamma^{2}+J_{0}^{2} \omega_{\mathrm{x}}+\omega_{\mathrm{z}}^{2}} .
$$

4. ETUde DÉTAillée du SIGNAL A LA FRÉQUENCE $\omega / 2 \pi$. - En raison des avantages qu'il présente, nous discutons plus en détail les caractéristiques du signal à la fréquence $\omega / 2 \pi$. C'est en effet le signal modulé en forme de dispersion que l'on peut obtenir avec l'intensité la plus grande. Pour cette raison, c'est lui qui a été utilisé dans le magnétomètre décrit dans l'introduction et que nous avons choisi pour effectuer les vérifications expérimentales des calculs précédents. Le niveau atomique étudié est l'état fondamental de ${ }^{87} \mathrm{Rb}$. Nous avons utilisé une cellule paraffinée, sans gaz tampon, dont le temps de relaxation est de l'ordre de $1 \mathrm{~s}$. Elle est placée dans un blindage magnétique qui élimine les fluctuations et les inhomogénéités du champ terrestre. Trois paires de bobines permettent de créer des champs magnétiques dans les trois directions. Pour une description détaillée du montage expérimental, on se reportera à la référence [2].

L'état fondamental de ${ }^{87} \mathrm{Rb}$ comprend deux niveaux hyperfins $F=2$ et $F=1$; les conditions expérimentales sont donc différentes de celles supposées dans le calcul. Une étude plus détaillée montre que seule l'échange d'orientation entre les deux niveaux hyperfins est susceptible d'introduire des phénomènes nouveaux. En particulier la forme des résonances doit être en principe très légèrement différente de celles prévues par le calcul précédent. La correction est cependant trop faible pour justifier une complication appréciable du modèle théorique simple présenté ici.

Le signal à la fréquence $\omega / 2 \pi$ s'écrit donc

$$
S=-\frac{1}{\Gamma}\left(\lambda J_{0}\right)\left(2 J_{1} \sin \omega t\right) s
$$

où

$$
s=\frac{\Gamma \bar{\omega}_{\mathrm{z}}+\bar{\omega}_{\mathrm{x}} \bar{\omega}_{\mathrm{y}}}{\Gamma^{2}+\bar{\omega}_{\mathrm{x}}^{2}+\bar{\omega}_{\mathrm{y}}^{2}+\bar{\omega}_{\mathrm{z}}^{2}}
$$

Rappelons que $\bar{\omega}_{\mathrm{z}}=\bar{\omega}_{\mathrm{z}}, \bar{\omega}_{\mathrm{x}}=\omega_{\mathrm{x}} J_{0}, \bar{\omega}_{\mathrm{y}}=\omega_{\mathrm{y}} J_{0}$.

- L'amplitude de la courbe de résonance dépend de $\omega_{1} / \omega$ par le facteur $J_{0} J_{1}$. Elle est maximale pour $\omega_{1} / \omega \approx 1,1$ et $\left(J_{0} J_{1}\right)$ vaut alors 0,34 .

- La forme de la courbe de résonance est donnée par l'expression de s.
Pour des champs $\mathrm{H}$ petits devant la largeur de raie $(|\gamma \mathrm{H}| \ll \Gamma)$, on peut développer l'expression de $\mathrm{s}$ au $2^{\mathrm{e}}$ ordre. On retrouve la formule

$$
\mathcal{S}=-2 \frac{\lambda}{\Gamma} J_{0} J_{1} \sin \omega t\left(\frac{\omega_{\mathrm{z}}}{\Gamma}+J_{0}^{2} \frac{\omega_{\mathrm{x}} \omega_{\mathrm{y}}}{\Gamma^{2}}\right),
$$

donnée dans la référence [2] (*). Le signal est au premier ordre proportionnel à $\omega_{\mathrm{z}}$ et ne dépend qu'au second ordre de $\omega_{\mathrm{x}}$ et $\omega_{\mathrm{y}}$.

$\mathrm{Si}$ on balaie $\omega_{\mathrm{z}}$, l'une seulement des autres composantes étant non nulle (par exemple $\omega_{\mathrm{y}}$ ), la courbe de résonance est une courbe de dispersion $\frac{\Gamma \bar{\omega}_{\mathrm{z}}}{\left(\Gamma^{2}+\bar{\omega}_{\mathrm{y}}^{2}\right)+\bar{\omega}_{\mathrm{z}}^{2}}$ comme dans la «résonance paramétrique ordinaire " mais élargie. La largeur de la courbe correspond maintenant à $\Delta \omega_{\mathrm{z}}=2 \sqrt{\Gamma^{2}+J_{0}^{2} \omega_{\mathrm{y}}^{2}}$.

La figure 3 montre la largeur des courbes expéri-

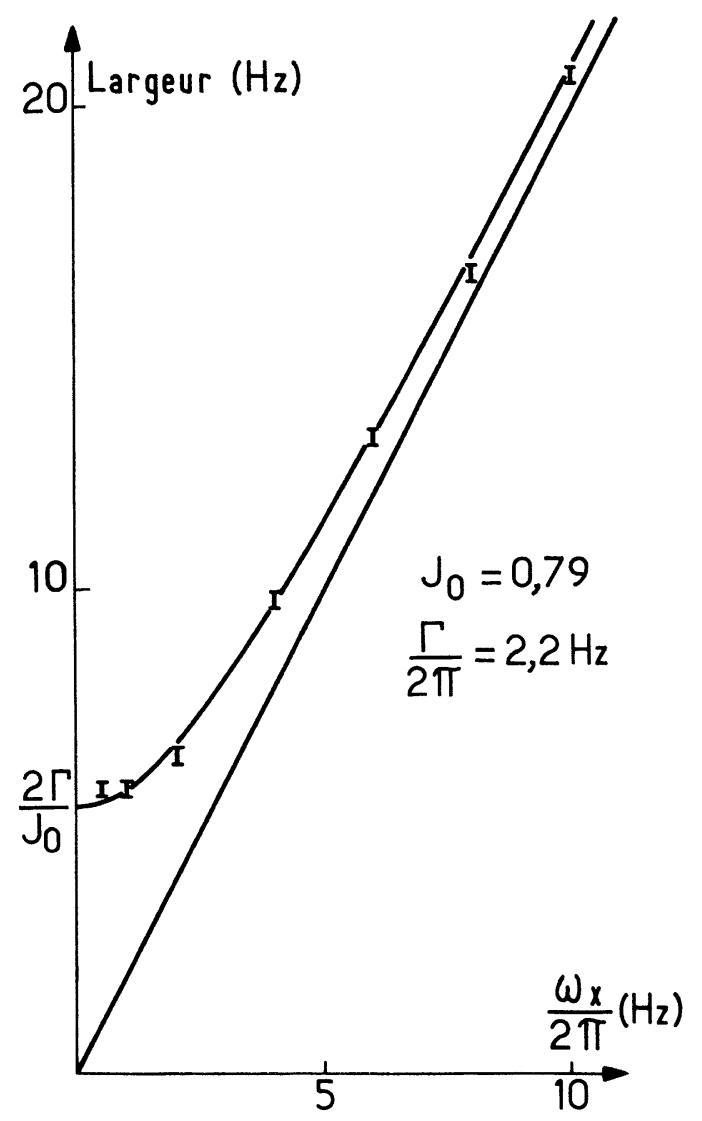

Fig. 3. - Largeur, en fonction d'une décompensation transverse $H_{\mathrm{y}}$, des résonances apparaissant lorsqu'on balaye $H_{\mathrm{z}}$.

Erratum : Sur la figure au lieu de $\frac{\omega_{\mathrm{x}}}{2 \pi}$ lire $\frac{\omega_{\mathrm{y}}}{2 \pi}$

mentales exprimée en $\mathrm{Hz}$ pour diverses valeurs $\omega_{\mathrm{y}} / 2 \pi$. La courbe théorique a été tracée pour les valeurs $J_{0}=0,79$ et $\Gamma / 2 \pi=2,2 \mathrm{~Hz}$ qui correspondaient aux valeurs expérimentales utilisées. La pente des courbes de résonance en $\omega_{z}=0$, qui mesure la sensibilité du magnétomètre est multipliée par

(*) Les signes de la référence [2] sont différents, car on y a posé $\omega_{\mathrm{x}}=\gamma H_{\mathrm{x}}, \ldots$ 


$$
\frac{1}{1+\left(J_{0} \omega_{y} / \Gamma\right)^{2}}
$$

facteur toujours inférieur à 1. La présence d'une décompensation transversale diminue donc la sensibilité du magnétomètre à un champ parallèle à $\mathrm{Oz}$. La figure 4 montre la variation du facteur de réduction de la sensibilité en fonction de $\omega_{\mathrm{y}} / 2 \pi$. La courbe est théorique et les points, expérimentaux.

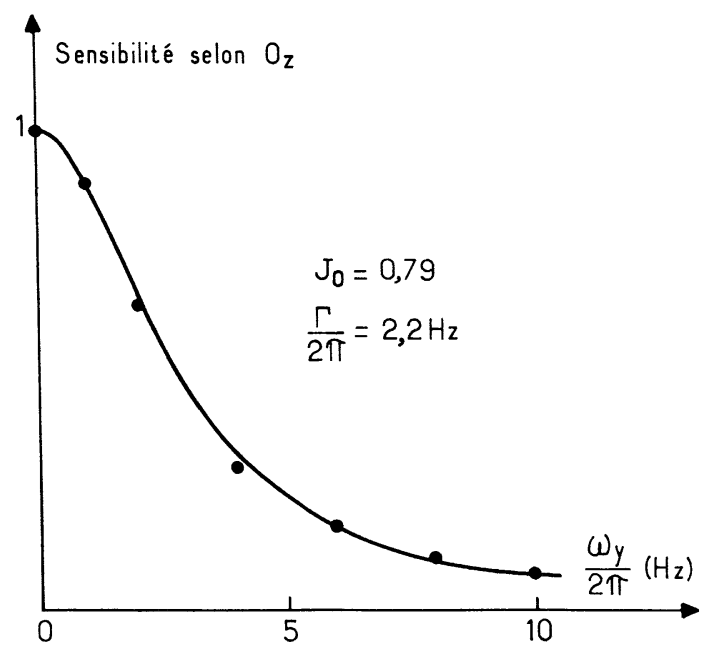

FIG. 4. - Sensibilité du magnétomètre à $H_{\mathrm{z}}$ en fonction d'une décompensation transverse.

Si maintenant, les deux composantes $\omega_{\mathrm{x}}$ et $\omega_{\mathrm{y}}$ sont non nulles, la courbe de résonance n'a plus la forme d'une dispersion pure. En particulier, le signal qui s'annulait pour $\omega_{\mathbf{z}}=0$, s'annule maintenant pour

$$
\bar{\omega}_{\mathrm{z}}=-\left(\frac{\bar{\omega}_{\mathrm{x}} \bar{\omega}_{\mathrm{y}}}{\Gamma}\right)
$$

L'annulation de $s$ ne repère donc plus le champ nul. Il faut toutefois remarquer que si on peut régler $\omega_{\mathrm{x}}$ et $\omega_{\mathrm{y}}$ à zéro à $1 / 100$ de la largeur de la résonance près, l'annulation de $s$ repère le zéro de $\omega_{\mathbf{z}}$ avec une erreur inférieure à $10^{-4}$ de la largeur.

Sur la figure 5 , on a porté en fonction de $\omega_{y}$, la valeur de $\omega_{z}$ pour laquelle $s$ s'annule, et ceci pour diverses valeurs de $\omega_{\mathrm{x}}$. Le réseau théorique est un ensemble de droites dont la pente est proportionnelle à $\omega_{\mathbf{x}}$. Les droites sont théoriques, tracées pour les valeurs $J_{0}=0,73$ et $\Gamma / 2 \pi=3,1 \mathrm{~Hz}$.

$\mathrm{Si}$ on balaye une composante perpendiculaire au champ de R. F., par exemple $H_{\mathrm{y}}, H_{\mathrm{z}}$ étant nul, on obtient pour $s$ une courbe de dispersion

$$
s=\frac{\vec{\omega}_{\mathrm{x}}}{\Gamma} \frac{\Gamma \vec{\omega}_{\mathrm{y}}}{\left(\Gamma^{2}+\bar{\omega}_{\mathrm{x}}^{2}\right)+\bar{\omega}_{\mathrm{y}}^{2}} .
$$

Son amplitude est $\frac{\bar{\omega}_{\mathrm{x}}}{\sqrt{\Gamma^{2}+\bar{\omega}_{\mathrm{x}}^{2}}}$ sa largeur

$\Delta \bar{\omega}_{\mathrm{y}}=2 \sqrt{\Gamma^{2}+\bar{\omega}_{\mathrm{x}}^{2}}$ soit

$$
\Delta \omega_{\mathrm{y}}=2 \sqrt{\omega_{\mathrm{x}}^{2}+\left(\Gamma / J_{0}\right)^{2}} .
$$

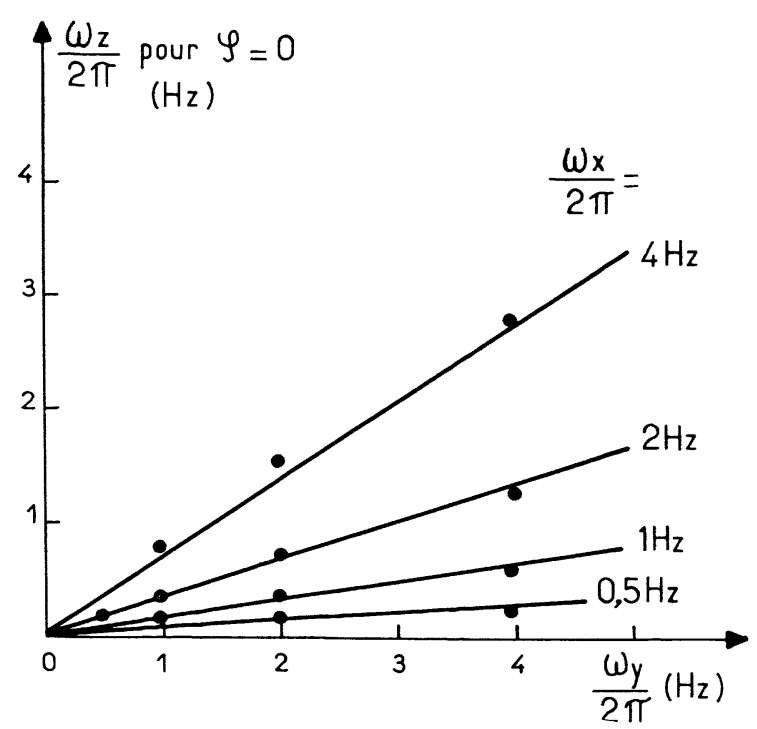

Fig. 5. - Déplacement du zéro du signal $\$$ de résonance paramétrique (modulation $\omega$ ) en fonction des décompensations transverses $H_{\mathrm{x}}$ et $\boldsymbol{H}_{\mathrm{y}}$.

Il n'y a aucun signal si $\omega_{\mathrm{x}}=0$. Mais si $\omega_{\mathrm{x}}$ est non nul, le signal en forme de dispersion apparaît, d'autant plus grand et plus large que $\omega_{\mathrm{x}}$ est grand. Lorsque $\omega_{\mathrm{x}} \gg \Gamma$, l'intensité tend vers 1 et sa largeur est égale à $\Delta \omega_{\mathrm{y}}=2 \omega_{\mathrm{x}}$.

La figure 6 montre la forme du signal pour diverses valeurs de $\omega_{\mathrm{x}} / 2 \pi$. La largeur et l'intensité sont comparées aux variations théoriques sur les figures 7 et 8 .

On peut se servir de ce signal pour annuler $\omega_{\mathrm{y}}$ : il suffit, $\omega_{\mathrm{x}}$ étant différent de zéro, d'annuler la modulation $\omega / 2 \pi$ de la lumière transmise. La sensibilité est proportionnelle à $\bar{\omega}_{\mathrm{x}} \Gamma / \Gamma^{2}+\bar{\omega}_{\mathrm{x}}^{2}$. Elle est maxi-

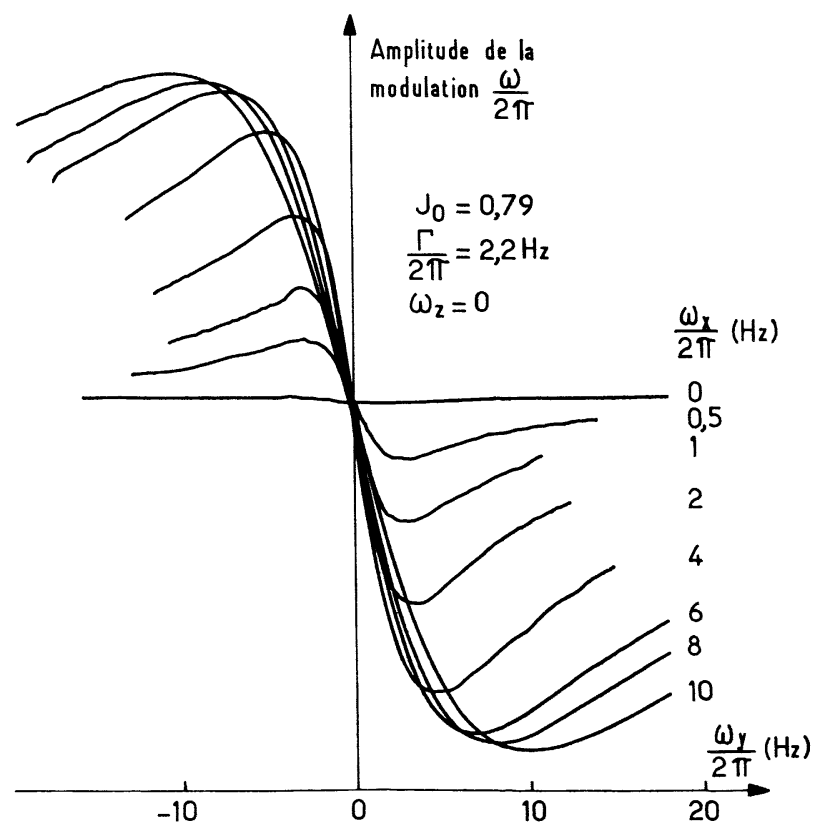

FIG. 6. - Résonances apparaissant lorsqu'on balaye $H_{\mathrm{y}}$, pour diverses valeurs de $\boldsymbol{H}_{\mathbf{x}}$. 

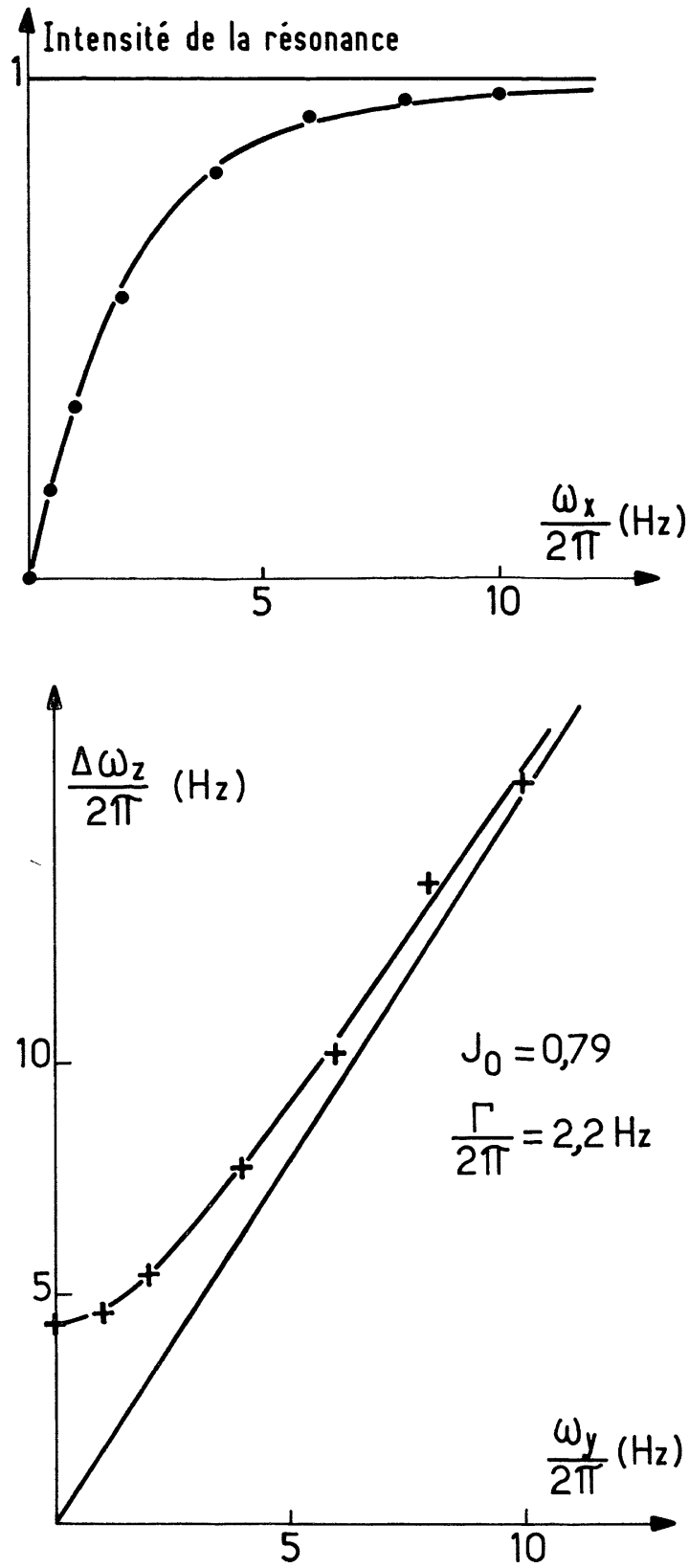

FIG. 7 et 8 . - Intensité et largeur de ces résonances en fonction de $H_{\mathrm{x}}$.

mum pour $\bar{\omega}_{\mathrm{x}}=\Gamma$. Pour avoir une sensibilité maximum selon Oy, il faut donc décompenser $\omega_{\mathrm{x}}$ de $\Gamma / J_{0}$. La sensibilité vaut alors, comparativement à celle $\mathrm{du}$ magnétomètre selon $\mathrm{Oz}, J_{0} / 2$.

On a reporté sur la figure 9 les sensibilités en $H_{\mathrm{y}}$, mesurées pour diverses valeurs de la décompensation en $H_{\mathrm{x}}$. La courbe est calculée à partir de la formule théorique (pour les valeurs $J_{0}=0,79, \Gamma / 2 \pi=2,2 \mathrm{~Hz}$ ).

Tous ces résultats demeurent valables lorsqu'on échange $H_{\mathrm{y}}$ et $H_{\mathrm{x}} ; \omega_{\mathrm{x}}$ et $\omega_{\mathrm{y}}$ jouent en effet des rôles symétriques dans la formule (11).

On dispose ainsi d'une méthode simple pour détecter des petites variations des composantes $H_{\mathrm{x}}$ et $H_{\mathrm{y}}$ du champ magnétique avec une sensibilité comparable à

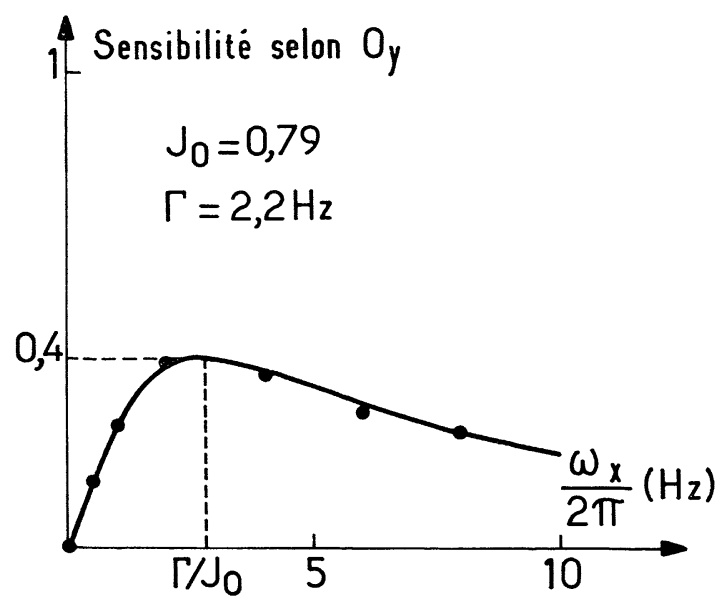

Fig. 9. - Sensibilité du magnétomètre à $H_{\mathrm{y}}$, en fonction de $H_{\mathbf{x}}$.

celle que donne la résonance paramétrique dans la direction $\mathrm{Oz}$.

$\mathrm{Si}$, par contre, $\omega_{\mathrm{z}}$ n'est pas nul, $\omega_{\mathbf{x}}$ ayant été décompensé de la quantité optimum $\Gamma / J_{0}$, la nullité de la modulation $\omega / 2 \pi$ se produit pour

$$
\bar{\omega}_{\mathrm{z}}+\frac{\bar{\omega}_{\mathrm{x}} \bar{\omega}_{\mathrm{y}}}{\Gamma}=0
$$

soit $\omega_{\mathrm{y}}=-\omega_{\mathrm{z}} / J_{0}$. Le zéro de la modulation $\omega / 2 \pi$ ne repère donc plus la nullité de $H_{\mathrm{y}}$, l'erreur commise étant de l'ordre de grandeur de $H_{\mathrm{z}}$.

5. MÉTHOde PRATIQUe DE COMPENSATION DES TROIS COMPOSANTES D'UN CHAMP MAGNÉTIQUE. - Indiquons maintenant comment on peut au moyen des résonances précédentes annuler dans une région de l'espace les trois composantes du champ magnétique. Si l'on utilise des atomes de ${ }^{87} \mathrm{Rb}$ contenus dans une cellule qui permet d'obtenir des temps de relaxation de l'ordre de la seconde, on peut réaliser cette annulation du champ avec une précision approchant $10^{-9} \mathrm{G}$. Supposons la cellule placée dans un champ magnétique inconnu. Trois paires de bobines en position de Helmholtz permettent de créer des champs dans les trois directions $\mathrm{Ox}, \mathrm{Oy}, \mathrm{Oz}$. Il s'agit de déterminer les courants dans les trois bobines qui compensent exactement le champ qui régnait initialement. La mesure de ces courants permet d'ailleurs de mesurer les trois composantes de ce champ magnétique.

a) Réglage préliminaire utilisant l'effet Hanle [10]. - Il permet de compenser le champ magnétique avec une précision de l'ordre de la largeur de raie. Rappelons brièvement le principe de ce réglage [11]: les atomes sont pompés selon l'axe $\mathrm{Oz}$; il n'y a aucun champ de radiofréquence. On peut montrer que l'intensité lumineuse absorbée par la cellule est alors proportionnelle à 


$$
M_{\mathrm{x}}=\frac{\lambda}{\Gamma} \frac{\Gamma^{2}+\omega_{\mathrm{x}}^{2}}{\Gamma^{2}+\omega_{\mathrm{x}}^{2}+\omega_{\mathrm{y}}^{2}+\omega_{\mathrm{z}}^{2}}
$$

qui n'est autre que la formule (3) où l'on a fait $\omega_{1}=0$. On commence par régler $\omega_{\mathrm{x}}$ de façon à ce qu'il $\mathrm{y}$ ait une orientation dans la cellule (c'est toujours possible puisque $M_{\mathrm{x}}$ vaut $\lambda / \Gamma$ pour $\left.\omega_{\mathrm{x}} \approx \infty\right)$. Les valeurs $\omega_{\mathrm{y}}$ et $\omega_{\mathrm{z}}$ nulles sont celles qui rendent le dénominateur minimum, donc $M_{\mathbf{x}}$ maximum. Ce premier réglage de $\omega_{y}$ et $\omega_{z}$ étant ainsi effectué, on décompense $H_{y}$ d'une quantité connue, supérieure à la largeur de raie : on a alors

$$
M_{\mathrm{x}}=\frac{\lambda}{\Gamma} \frac{\Gamma^{2}+\omega_{\mathrm{x}}^{2}}{\Gamma^{2}+\omega_{\mathrm{x}}^{2}+\omega_{\mathrm{y}}^{2}} ;
$$

on règle ensuite $\omega_{\mathbf{x}}$ à la valeur 0 en cherchant la valeur de $H_{\mathrm{x}}$ qui rend $M_{\mathrm{x}}$ minimum. Puis on recompense $H_{\mathrm{y}}$. Le réglage d'une composante est d'autant plus précis que les autres sont voisines de zéro, de sorte que plusieurs cycles de réglages successifs sont nécessaires pour parvenir à la meilleure compensation possible. Les 3 composantes résiduelles $H_{\mathrm{x}}^{0}, H_{\mathrm{y}}^{0}, H_{\mathrm{z}}^{0}$ du champ sont alors petites devant la largeur :

Posons

$$
\mathrm{x}_{0}=\frac{\bar{\omega}_{\mathrm{x}}^{0}}{\Gamma}, \mathrm{y}_{0}=\frac{\bar{\omega}_{\mathrm{y}}^{0}}{\Gamma}, \mathrm{z}_{0}=\frac{\bar{\omega}_{\mathrm{z}}^{0}}{\Gamma}
$$

on a $\mathrm{x}_{0}, \mathrm{y}_{0}, \mathrm{z}_{0} \ll 1$.

b) Réglages fins utilisant les résonances paramétriques. - On introduit maintenant le champ de R. F. $\mathbf{H}_{1} \cos \omega t$ dans la direction $\mathrm{Oz}$ : une modulation à la fréquence $\omega / 2 \pi$ apparaît sur la lumière transmise car le réglage précédent n'étant pas très précis, le champ n'est pas exactement compensé. On annule cette modulation en réglant $H_{\mathrm{z}}$; d'après l'expression du signal

$$
s=\frac{z+x y}{1+x^{2}+y^{2}+z^{2}}
$$

où $\mathrm{x}=\bar{\omega}_{\mathrm{x}} / \Gamma, \mathrm{y}=\bar{\omega}_{\mathrm{y}} / \Gamma, \mathrm{z}=\bar{\omega}_{\mathrm{z}} / \Gamma, \mathrm{z}$ vaut alors $\mathrm{z}_{1}=-\mathrm{x}_{0} \mathrm{y}_{0}$. Décompensons $H_{\mathrm{y}}$ d'une quantité de l'ordre de la largeur $(\mathrm{y} \approx 1)$. La modulation réapparaît. On l'annule en réglant $H_{\mathrm{x}}: \mathrm{x}$ vaut alors d'après la formule précédente $\mathrm{x}_{1} \approx-\mathrm{z}_{1}=+\mathrm{x}_{0} \mathrm{y}_{0}$. On recompense $H_{\mathrm{y}}$. On décompense $H_{\mathrm{x}}$ d'une quantité de l'ordre de la largeur : on annule la modulation en réglant $H_{\mathrm{y}}$; ce qui donne $\mathrm{y}=\mathrm{y}_{1} \approx-\mathrm{z}_{1}=\mathrm{x}_{0} \mathrm{y}_{0}$. On supprime enfin la décompensation de $H_{\mathrm{x}}$.

On recommence ensuite le cycle de réglage à partir des valeurs $\mathrm{x}_{1}, \mathrm{y}_{1}, \mathrm{z}_{1}$ précédemment obtenues et qui sont inférieures aux valeurs initiales. Au $n_{\text {ième cycle de }}$ réglage, on obtient $\mathrm{z}_{n}=-\mathrm{x}_{n-1} \mathrm{y}_{n-1} ; \mathrm{x}_{n} \approx \mathrm{y}_{n} \approx-\mathrm{z}_{n}$ d'où $\mathrm{z}_{n} \approx-\left(\mathrm{z}_{n-1}\right)^{2}$. Soit encore

$$
\left|\mathrm{x}_{n}\right| \approx\left|\mathrm{y}_{n}\right| \approx\left|\mathrm{z}_{n}\right| \approx\left|\mathrm{z}_{0}\right|^{\left(2^{n}\right)} \text {. }
$$

On constate donc que $\left|\mathrm{x}_{n}\right|,\left|\mathrm{y}_{n}\right|,\left|\mathrm{z}_{n}\right|$ décroissent très rapidement avec $n$. Expérimentalement, quelques cycles de réglages suffisent en général pour arriver à la limite de sensibilité de l'appareil pourvu qu'au départ les trois composantes du champ soient inférieures à la largeur de raie. Le réglage que nous venons de décrire est plus précis que celui utilisant seulement l'effet Hanle pour deux raisons :

Tout d'abord, le signal apparaît sur une modulation, donc le rapport signal/bruit est considérablement meilleur. De plus, les signaux qu'on observe sont proportionnels aux décompensations (forme de courbe en dispersion) alors que dans l'autre procédé, ils étaient du deuxième ordre (courbe en absorption).

On peut utiliser une autre méthode pour régler les champs $H_{\mathrm{x}}$ et $H_{\mathrm{y}}$. Supposons $H_{\mathrm{z}}$ réglé :

$$
\omega_{\mathrm{z}}=0, s=\frac{\bar{\omega}_{\mathrm{x}} \bar{\omega}_{\mathrm{y}}}{\Gamma^{2}+\bar{\omega}_{\mathrm{x}}^{2}+\bar{\omega}_{\mathrm{v}}^{2}} .
$$

Si on change brusquement $H_{\mathrm{y}}$ d'une quantité connue $\delta H_{\mathrm{y}}$, le signal $s$ varie d'une quantité $\delta s$ qui est proportionnelle à $\omega_{\mathrm{x}}$ : en particulier si $\omega_{\mathrm{x}}=0, s$ ne varie pas. L'insensibilité du signal à une variation de $H_{\mathrm{y}}$ est donc un critère permettant de savoir que $H_{\mathrm{x}}$ est réglé. $H_{\mathrm{x}}$ et $H_{\mathrm{y}}$ jouant dans la formule des rôles symétriques, on peut aussi régler $H_{\mathrm{y}}$ en faisant varier $H_{\mathrm{x}}$.

Si $H_{z}$ n'est pas tout à fait nul, ceci n'est plus exact : le signal est insensible à une variation de $H_{\mathrm{y}}$ pour une certaine valeur de $H_{\mathrm{x}}$, de l'ordre de grandeur de $H_{z}$. Mais de même que dans l'autre méthode, plusieurs réglages successifs conduisent rapidement à la compensation exacte.

II. Résonances en champ nul en présence de deux champs de radiofréquence. - En utilisant les résultats précédents, nous dégageons maintenant l'idée d'une méthode différente, permettant d'obtenir des signaux indépendants respectivement proportionnels (au moins en champ faible, $|\gamma H|<\Gamma$ ) aux trois composantes du champ magnétique.

1. IDÉE GÉNÉRALE DE LA MÉTHODE. - Reprenons le même dispositif expérimental que précédemment. Nous avons montré $(1,5, b)$ que, lorsqu'on décompense $H_{\mathrm{y}}$, l'amplitude $s$ du signal à la fréquence $\omega / 2 \pi$ varie d'une quantité $\delta s$, proportionnelle à $H_{\mathrm{x}}$. Si donc on ajoute parallèlement à $\mathrm{Oy}$ un champ oscillant $\mathfrak{H}_{1} \cos \Omega t$, l'amplitude $s$ de la modulation $\omega$ de la lumière transmise va être elle-même modulée à la fréquence $\Omega$, l'amplitude de cette modulation (en $\sin \omega t \cos \Omega t$ ) étant proportionnelle à $H_{\mathbf{x}}$ (la «bande passante » atomique étant de l'ordre de $\Gamma, \Omega$ ne doit pas être trop grand devant $\Gamma$; nous reviendrons sur ce point dans la suite). Par ailleurs, il subsiste une modulation en $\sin \omega t$ (résonance paramétrique dans le champ $H_{1} \cos \omega t$, parallèle à $\mathrm{Oz}$ ) dont l'amplitude varie avec $H_{\mathrm{z}}$ comme une courbe de dispersion. Enfin, le champ oscillant $\mathscr{H}_{1} \cos \Omega t$, parallèle à Oy, produit le phénomène de résonance paramétrique, et la lumière transmise doit être également modulée à la fréquence 
$\Omega / 2 \pi$ (et tous ses harmoniques), l'amplitude de cette modulation variant comme une courbe de dispersion en fonction de $H_{\mathbf{y}}$. On obtient ainsi simultanément sur la lumière transmise 3 modulations distinctes (respectivement en $\sin \omega t \cos \Omega t, \sin \omega t, \sin \Omega t$ ), dont les amplitudes sont proportionnelles en champ faible, aux trois composantes du champ magnétique.

En fait, on ne se trouve pas dans le cas d'une modulation lente du champ $H_{\mathrm{y}}$, qui correspondrait à $\Omega \ll \Gamma$. L'amplitude de la modulation en $\sin \omega t \cos \Omega t$ ne peut donc pas s'obtenir à partir de la formule (10) en remplaçant simplement $\omega_{\mathrm{y}}$ par $\omega_{\mathrm{y}}+\Omega_{1} \cos \Omega t$ $\left(\Omega_{1}=-\gamma \mathcal{H C}_{1}\right)$. Comme d'autre part la condition $\Omega \gg \Gamma$ de la résonance paramétrique n'est pas exactement remplie pour le $2^{\mathrm{e}}$ champ de radiofréquence, le signal de résonance en $\sin \Omega t$ ne sera pas exactement celui d'une résonance paramétrique. Enfin, il est inexact de considérer que les deux champs de R. F. $\mathbf{H}_{1} \cos \omega t$ et $\mathfrak{H}_{1} \cos \Omega t$ agissent indépendamment sur l'atome. Une théorie des résonances en champ nul en présence de deux champs de R. F. est donc nécessaire pour confirmer la discussion qualitative précédente relative à l'existence des 3 modulations distinctes, proportionnelles aux 3 composantes du champ. Ce sont les résultats de cette théorie et leur vérification expérimentale que nous présentons dans cette deuxième partie.

Dans le $\S 2$, nous exposons le principe de 2 méthodes de calcul différentes de la forme des signaux. Nous discutons au $\S 3$ les résultats obtenus dont certains s'interprètent de façon simple à la lumière des quelques notions sur "l'atome habillé " rappelées dans la partie I. Les vérifications expérimentales sont décrites au fur et à mesure.

2. PRinCiPes GÉNÉRAuX DeS CAlCUlS et RÉSUltats. - Les atomes pompés optiquement dans la direction Ox sont donc soumis à l'action de deux champs de radiofréquence $\mathbf{H}_{1} \cos \omega t$ parallèle à $\mathrm{Oz}$ et $\mathfrak{H}_{1} \cos \Omega t$ parallèle à $\mathrm{Oy}$, en présence d'un champ statique $\mathbf{H}$ de direction quelconque (Fig. 10).

Nous ferons les hypothèses suivantes:

$$
\Gamma<\Omega \ll \omega
$$

$\Omega / \Gamma \approx 5$ à 10 (nous verrons plus loin les raisons qui guident ce choix).

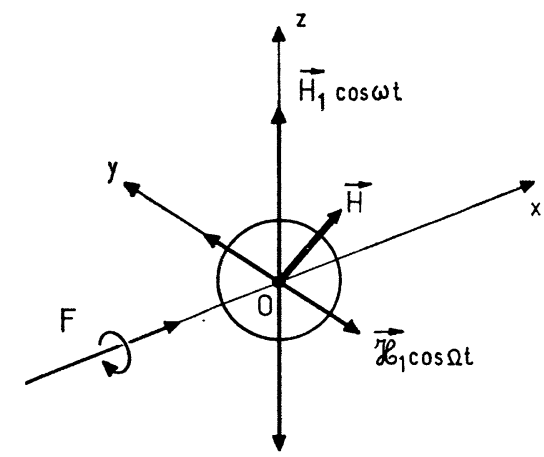

FIG. 10. - Résonance paramétrique avec 2 champs de R.F. $\omega_{1}=-\gamma H_{1}$ et $\Omega_{1}=-\gamma \boldsymbol{H}_{1}$ sont respectivement inférieures ou de l'ordre de grandeur de $\omega$ et $\Omega$.

a) Première méthode. - Les champs $\mathbf{H}$ et $\boldsymbol{H}_{1} \cos \Omega t$ sont traités comme une perturbation affectant le mouvement de l'orientation sous l'action de $\mathbf{H}_{1} \cos \omega t$. Nous nous limiterons donc dans ce premier calcul à des amplitudes faibles de $\mathbf{H}$ et $\mathscr{H}_{1}: \omega_{\mathbf{x}} / \Gamma$, $\omega_{\mathrm{y}} / \Gamma, \omega_{\mathrm{z}} / \Gamma, \Omega_{1} / \Omega \ll 1$. La méthode de calcul utilisée est la même que celle de la première partie. On remplace seulement dans les équations (1) $\omega_{\mathrm{y}}$ par

$$
\omega_{\mathrm{y}}+\Omega_{1} \cos \Omega t
$$

La présence du terme modulé $\Omega_{1} \cos \Omega t$ complique les calculs de sorte que nous nous contenterons des termes jusqu'au $3^{\mathrm{e}}$ ordre en $\omega_{\mathrm{x}} / \Gamma, \omega_{\mathrm{y}} / \Gamma, \omega_{\mathrm{z}} / \Gamma, \Omega_{1} / \Omega$ et d'ordre 0 et 1 en $\Gamma / \Omega$.

On trouve sur la lumière transmise des signaux modulés, parmi lesquels nous retiendrons :

une modulation à la fréquence $\omega$

$-\left(\frac{\lambda J_{0}}{\Gamma}\right)\left(2 J_{1} \sin \omega t\right) \times$

$\left.\times\left\{\frac{\bar{\omega}_{\mathrm{z}}}{\Gamma}\left[1-\frac{\bar{\omega}_{\mathrm{z}}^{2}+\bar{\omega}_{\mathrm{x}}^{2}+\bar{\omega}_{\mathrm{y}}^{2}}{\Gamma^{2}}\right]-2\left(\frac{\bar{\Omega}_{1}}{2 \Omega}\right)^{2}\right)+\frac{\bar{\omega}_{\mathrm{x}} \bar{\omega}_{\mathrm{y}}}{\Gamma^{2}}\right\}$,

une modulation à la fréquence $\Omega$

$$
\begin{aligned}
&-\left(\frac{\lambda J_{0}}{\Gamma}\right)\left(2 J_{0}\right.\left.\frac{\bar{\Omega}_{1}}{2 \Omega} \sin \Omega t\right)\left\{\frac{\bar{\omega}_{\mathrm{y}}}{\Gamma}-\frac{\bar{\omega}_{\mathrm{x}} \bar{\omega}_{\mathrm{z}}}{\Gamma^{2}}\right\}+ \\
&+\left(2 \frac{\lambda J_{0}}{\Gamma}\right)\left(J_{0} \frac{\bar{\Omega}_{1}}{2 \Omega} \cos \Omega t\right) \frac{\Gamma}{\Omega} \frac{\bar{\omega}_{\mathrm{x}} \bar{\omega}_{\mathrm{z}}}{\Gamma^{2}}
\end{aligned}
$$

un signal à la fréquence $\omega$, modulé à $\Omega$

$$
\begin{aligned}
\left(\frac{\lambda J_{0}}{\Gamma}\right)\left(2 J_{1} \sin \omega t\right)\left(2 \frac{\bar{\Omega}_{1}}{2 \Omega}\right. & \cos \omega t) \times \\
\times & \frac{\Gamma}{\Omega}\left\{\frac{\bar{\omega}_{\mathrm{x}}}{\Gamma}-\frac{\bar{\omega}_{\mathrm{z}} \bar{\omega}_{\mathrm{x}}}{\Gamma^{2}}\right\} .
\end{aligned}
$$

Rappelons que $J_{n}$ est mis pour $J_{n}\left(\omega_{1} /\right)\left(J_{n}\right.$ fonction de Bessel d'ordre $n$ ), $\bar{\omega}_{\mathrm{x}}=J_{0} \omega_{\mathrm{x}}, \bar{\omega}_{\mathrm{y}}=J_{0} \omega_{\mathrm{y}}, \bar{\omega}_{\mathrm{z}}=\omega_{\mathrm{z}}$. Nous posons de même $\Omega_{1}=\bar{\Omega}_{1} J_{0}$.

b) Deuxième méthode. - On traite quantiquement les deux champs de radiofréquence en utilisant «le formalisme de l'atome habillé » [9]. Le problème consiste alors à étudier les variations de la lumière absorbée par le système total « atome +2 champs de radiofréquence» lorsqu'on balaie le champ magnétique statique $\mathbf{H}$ dans une direction quelconque autour de la valeur zéro.

Deux approximations sont faites : on néglige les termes $\Omega_{1} / \omega$ et $\Omega / \omega$. Nous avons en effet supposé $\Omega \ll \omega$ et $\Omega_{1} \approx \Omega$. On ne tient, d'autre part, compte qu'aux ordres 0 et 1 des termes en $\Gamma / \Omega$. Ces termes interviennent du fait que le champ $\mathfrak{H}_{1} \cos \Omega t$ ne vérifie 
pas bien la condition de résonance paramétrique $\Omega \gg \Gamma$. Leur importance sera d'autant plus grande (et cette approximation d'autant moins justifiée) qu'on aura choisi $\Omega$ plus près de $\Gamma$.

Par contre, et c'est là ce qui constitue la supériorité de cette méthode sur la précédente, l'effet des champs $\mathrm{H}$ et $\mathscr{H}_{1} \cos \Omega t$ est traité rigoureusement à tous les ordres en $\gamma H / \Gamma$ et $\Omega_{1} / \Omega$ (nous n'imposons donc plus ici les conditions $\gamma H \ll \Gamma$ et $\Omega_{1} \ll \Omega$ ). Les calculs peuvent être menés jusqu'au bout. Ceci montre la puissance du formalisme de "l'atome habillé». En effet, la lourdeur du calcul par itération rend impossible un calcul à un ordre supérieur à 3 en $\gamma H / \Gamma$, alors que la seconde méthode donne directement la resommation de toute la série de perturbation. Pour le détail des calculs, on se reportera à [6]. On obtient ainsi :

- un signal à la fréquence $\omega / 2 \pi$ :

$$
-\left(\lambda J_{0} \gamma_{0}\right)\left(2 J_{1} \sin \omega t\right) \frac{\gamma_{0} \omega_{\mathrm{z}}+\gamma_{0} J_{0}^{2} \frac{\omega_{\mathrm{x}} \omega_{y}}{\Gamma}}{\overline{\bar{\Delta}}}
$$

- un signal à la fréquence $\Omega / 2 \pi$

$$
\begin{array}{r}
-\left(\lambda J_{0} \check{\gamma}_{0}\right)\left(2 J_{0} \tilde{\gamma}_{1} \sin \Omega t\right) \frac{J_{0} \omega_{\mathrm{y}}-\check{\gamma}_{0}^{2} J_{0} \frac{\omega_{\mathrm{x}} \omega_{\mathrm{z}}}{\Gamma}}{\overline{\bar{\Delta}}}+ \\
+\frac{\Gamma}{\Omega} \cos \Omega t F\left(\omega_{\mathrm{x}}, \omega_{\mathrm{y}}, \omega_{\mathrm{z}}, \omega_{1}, \Omega_{1}\right)
\end{array}
$$

un signal à la fréquence $\omega \backslash 2 \pi$ d'amplitude modulée à $\Omega / 2 \pi$

$$
\begin{aligned}
& \left(\lambda J_{0}\right)\left(2 J_{1} \sin \omega t\right)\left(2 \tilde{\gamma}_{1} \cos \Omega t\right) \frac{\Gamma}{\Omega} \times \\
& \times \frac{\tilde{\gamma}_{0} J_{0} \omega_{\mathrm{x}}\left(1+\tilde{\gamma}_{0}^{2} \frac{\omega_{\mathrm{z}}^{2}+J_{0}^{2} \omega_{\mathrm{x}}^{2}}{\Gamma^{2}}\right)-\gamma_{0} J_{0} \frac{\omega_{\mathrm{y}} \omega_{\mathrm{z}}}{\Gamma}}{\overline{\bar{\Delta}}}
\end{aligned}
$$

où

$$
\overline{\bar{\Delta}}=\left(\gamma_{0} \bar{J}_{0} \omega_{\mathrm{x}}\right)^{2}+\left(J_{0} \omega_{\mathrm{y}}\right)^{2}+\left(\mathcal{F}_{0} \omega_{\mathrm{z}}\right)^{2}+\Gamma^{2} .
$$

$J_{n}$ est mis pour $J_{n}\left(\omega_{1} / \omega\right)$ ( $J_{n}$ fonction de Bessel d'ordre $n$ ) et $\gamma_{n}$ pour $J_{n}\left(\Omega_{1} / \Omega\right)$, valeur de la fonction de Bessel d'ordre $n$ pour l'argument

$$
\frac{\bar{\Omega}_{1}}{\Omega}=J_{0}\left(\frac{\omega_{1}}{\omega}\right) \frac{\Omega_{1}}{\Omega} .
$$

$F$, une fonction de $\omega_{\mathrm{x}}, \omega_{\mathrm{y}}, \omega_{\mathrm{z}}, \omega_{1} \Omega_{1}$, entièrement calculable. Nous ne donnons pas ici son expression explicite, car le signal correspondant est en quadrature avec celui en sin $\Omega t$ qui seul nous intéressera et qu'on peut isoler aisément au moyen d'une détection synchrone. Nous négligerons donc ce terme dans toute la suite.

En posant $\overline{\bar{\omega}}_{\mathrm{x}}=J_{0} \gamma_{0} \omega_{\mathrm{x}}, \overline{\bar{\omega}}_{\mathrm{y}}=J_{0} \omega_{\mathrm{y}}, \overline{\bar{\omega}}_{\mathrm{z}}=\gamma_{0} \omega_{\mathrm{z}}$, les trois signaux s'écrivent simplement :

$$
-\left(\lambda J_{0} \gamma_{0}\right)\left(2 J_{1} \sin \omega t\right) \frac{\bar{\omega}_{\mathrm{z}}+\frac{\overline{\bar{\omega}}_{\mathrm{x}} \bar{\omega}_{y}}{\Gamma}}{\overline{\bar{\Delta}}}
$$

$$
-\left(\lambda J_{0} \ddot{\gamma}_{0}\right)\left(2 J_{0} \tilde{\gamma}_{1} \sin \Omega t\right) \frac{\bar{\omega}_{\mathrm{y}}-\frac{\overline{\bar{\omega}}_{\mathrm{x}} \overline{\bar{\omega}}_{\mathrm{z}}}{\Gamma}}{\overline{\bar{\Delta}}}
$$

$\left(\lambda J_{0}\right)\left(2 J_{1} \sin \omega t\right)\left(2 \tilde{\gamma}_{1} \cos \Omega t\right) \frac{\Gamma}{\Omega} \times$

$$
\begin{gathered}
\times \frac{\overline{\bar{\omega}}_{\mathrm{x}}\left(1+\frac{\overline{\bar{\omega}}_{\mathrm{z}}^{2}+\overline{\bar{\omega}}_{\mathrm{x}}^{2}}{\Gamma^{2}}\right)-\frac{\overline{\bar{\omega}}_{\mathrm{y}} \overline{\bar{\omega}}_{\mathrm{z}}}{\Gamma}}{\overline{\bar{\Delta}}} \\
\overline{\bar{\Delta}}=\overline{\bar{\omega}}_{\mathrm{x}}^{2}+\overline{\bar{\omega}}_{\mathrm{y}}^{2}+\overline{\bar{\omega}}_{\mathrm{z}}^{2}+\Gamma^{2} .
\end{gathered}
$$

3. Discussion DES RÉSUltats. - $a$ ) En développant ces formules à l'ordre 3 en $\omega_{\mathrm{x}} / \Gamma, \omega_{\mathrm{y}} / \Gamma, \omega_{\mathrm{z}} / \Gamma$ et $\Omega_{1} / \Omega$, on retrouve les formules approchées données précédemment. Rappelons que

$$
J_{1}(\mathrm{x}) \approx \mathrm{x} / 2, J_{0}(\mathrm{x}) \approx 1-(\mathrm{x} / 2)^{2} \text { pour } \mathrm{x} \ll 1 .
$$

b) Dans les expressions des signaux, $H_{\mathrm{x}}, H_{\mathrm{y}}, H_{\mathrm{z}}$ ne figurent que par l'intermédiaire de $\overline{\bar{\omega}}_{\mathrm{x}}, \overline{\bar{\omega}}_{\mathrm{y}}, \overline{\bar{\omega}}_{\mathrm{z}}$. Ceci s'explique aisément: ces quantités représentent en effet les fréquences de Larmor de l'atome « habillé » successivement par les deux champs $\mathbf{H}_{1} \cos \omega t$ et $\mathfrak{H}_{1} \cos \Omega t$. Le premier, $\mathbf{H}_{1} \cos \omega t$, ne change pas $\omega_{\mathrm{z}}$ et multiplie $\omega_{\mathrm{x}}$ et $\omega_{\mathrm{y}}$ par $J_{0}\left(\omega_{1} / \omega\right)$. Il faut ensuite considérer l'effet du second champ $\mathfrak{H}_{1} \cos \Omega t$, dont la fréquence et l'amplitude sont petites par rapport à $\omega$; il agit non pas sur l'atome nu, mais sur l'atome habillé par le premier champ : comme il est dirigé selon $\mathrm{Oy}$, son amplitude intervient toujours multipliée par le facteur $J_{0}$, sous la forme $\bar{\Omega}_{1}=\Omega_{1} J_{0}$. Ce $2^{\mathrm{e}}$ champ modifie à son tour les fréquences de Larmor $\bar{\omega}_{\mathrm{x}}, \bar{\omega}_{\mathrm{y}}, \bar{\omega}_{\mathrm{z}}$ de l'atome habillé par $\mathbf{H}_{1} \cos \omega t$ : $\bar{\omega}_{\mathrm{x}}$ et $\bar{\omega}_{\mathrm{z}}$ sont remplacés par $\bar{\omega}_{\mathrm{x}} \gamma_{0}$ et $\bar{\omega}_{\mathrm{z}} \gamma_{0} ; \bar{\omega}_{\mathrm{y}}$ n'est pas changé. Les fréquences de Larmor de l'atome en présence des 2 champs de R. F. seront donc

$$
\overline{\bar{\omega}}_{\mathrm{x}}=\omega_{\mathrm{x}} J_{0} \gamma_{0}, \overline{\bar{\omega}}_{\mathrm{y}}=\omega_{\mathrm{y}} J_{0}, \overline{\bar{\omega}}_{\mathrm{z}}=\omega_{\mathrm{z}} \gamma_{0} .
$$

c) A l'ordre zéro en $\Gamma / \Omega$, la forme des signaux à $\omega$ et $\Omega$ est très semblable à celle du signal de résonance paramétrique étudié dans la partie I. Deux différences peuvent toutefois être notées, outre le remplacement des $\bar{\omega}_{\mathrm{x}, \mathrm{y}, \mathrm{z}}$ par les $\overline{\bar{\omega}}_{\mathrm{x}, \mathrm{y}, \mathrm{z}}$. La première est la présence dans le terme d'excitation de deux fonction de Bessel : le pompage est en effet perpendiculaire aux deux champs de radiofréquence, et son efficacité est multipliée par $J_{0}$ et $\gamma_{0}$. La deuxième est la modification des facteurs de détection des modulations $\omega$ et $\Omega$, le facteur de $\sin \omega t$ est $J_{1}$, celui de $\sin \Omega t$ est $\gamma_{1} J_{0}$. Ces deux termes peuvent s'interpréter entièrement dans le cadre de la théorie présentée dans la référence [9]. On peut donc finalement considérer que les deux champs de radiofréquence produisent indépendamment leurs signaux de résonance paramétrique, au changement des fréquences de Larmor et des facteurs de détection près. Ceci n'est pas vrai à l'ordre 1 en $\Gamma / \Omega$ puisqu'apparaît alors le signal 
en $\sin \omega t \cos \Omega t$ dont l'amplitude est un champ faible proportionnel à $\omega_{\mathbf{x}}$. Le fait que $\Omega$ n'est/pas très grand devant $\Gamma$, se traduit également ${ }_{\text {p }}$ ar une modification du signal à la fréquence $\Omega$ (apparition du terme "en $\cos \Omega t$ que nous avons négligé).

d) $\mathrm{Si}$ on se limite à $\gamma H \ll \Gamma$, on peut développer les signaux au $2^{\mathrm{e}}$ ordre en $\overline{\bar{\omega}}_{\mathrm{x}} / \Gamma, \overline{\bar{\omega}}_{\mathrm{y}} / \Gamma, \overline{\bar{\omega}}_{\mathrm{z}} / \Gamma$, tout en gardant les expressions rigoureuses en $\bar{\Omega}_{1} / \Omega$. Rappelons que la $1^{\text {re }}$ méthode de calcul exposée en $(2 a)$ conduit à des expressions qui ne sont valables qu'aux ordres les plus bas en $\bar{\Omega}_{1} / \Omega$. Ici nous avons en fait resommé toute la série de perturbations en $\bar{\Omega}_{1} / \Omega$, ce qui donne la dépendance exacte en $\bar{\Omega}_{1} / \Omega$, aussi bien des facteurs d'excitation et de détection $\left(\mathcal{\gamma}_{0}\right.$ ou $\left.\gamma_{1}\right)$, que des fréquences de Larmor modifiées $\left(\overline{\bar{\omega}}_{\mathbf{x}}\right.$, $\left.\overline{\bar{\omega}}_{\mathbf{y}},{ }^{i} \overline{\bar{\omega}}_{\mathbf{z}}\right)$. On obtient ainsi pour les 3 modulations :

$$
\begin{aligned}
& -\left(\frac{\lambda}{\Gamma} J_{0} \gamma_{0}\right)\left(2 J_{1} \sin \omega t\right)\left(\frac{\overline{\bar{\omega}}_{\mathrm{z}}}{\Gamma}+\frac{\overline{\bar{\omega}}_{\mathrm{x}} \overline{\bar{\omega}}_{\mathrm{y}}}{\Gamma^{2}}\right) \\
& -\left(\frac{\lambda}{\Gamma} J_{0} \tilde{\gamma}_{0}\right)\left(2 J_{0} \tilde{\gamma}_{1} \sin \omega t\right)\left[\frac{\bar{\omega}_{\mathrm{y}}}{\Gamma}-\frac{\overline{\bar{\omega}}_{\mathrm{x}} \overline{\bar{\omega}}_{\mathrm{z}}}{\Gamma^{2}}\right] \\
& \left(\frac{\lambda}{\Gamma} J_{0}\right)\left(2 J_{1} \sin \omega t\right)\left(2 \tilde{\gamma}_{1} \cos \Omega t\right) \frac{\Gamma}{\Omega}\left(\frac{\overline{\bar{\omega}}_{\mathrm{x}}}{\Gamma}-\frac{\overline{\bar{\omega}}_{\mathrm{y}} \overline{\bar{\omega}}_{\mathrm{z}}}{\Gamma^{2}}\right) .
\end{aligned}
$$

Chacun des 3 signaux est au premier ordre proportionnel à une des composantes du champ, et au $2^{\mathrm{e}}$ ordre, fonction du produit des deux autres composantes. Donc, au premier ordre les 3 signaux sont indépendants, ce que nous avons effectivement vérifié expérimentalement.

e) Les sensibilités au premier ordre sont dans les directions $\mathrm{Oz}, \mathrm{Oy}, \mathrm{Ox}$, respectivement proportionnelles à

$\sigma_{\mathrm{z}}=J_{0} J_{1} \check{\gamma}_{0}^{2}, \sigma_{\mathrm{y}}=J_{0}^{3} \tilde{\gamma}_{0} \tilde{\jmath}_{1}, \sigma_{\mathrm{x}}=2 J_{0}^{2} J_{1} \tilde{\gamma}_{0} \check{\partial}_{1} \frac{\Gamma}{\Omega}$.

Elles sont fonctions de $\omega_{1} / \omega, \Omega_{1} / \Omega$ et $\Gamma / \Omega$. Ce son ces dépendances que nous avons cherché à vérifier expérimentalement.

Les variations de $\sigma_{\mathrm{x}}, \sigma_{\mathrm{y}}, \sigma_{\mathrm{z}}$ en fonction de $\omega_{1} / \omega$ sont dues d'une part aux facteurs $J_{0}$ et $J_{1}$, d'autre part à la présence, dans $\gamma_{0}$ et $\gamma_{1}$ du facteur $J_{0}\left(\omega_{1} / \omega\right)$ devant $\Omega_{1} / \Omega$.

Cette dernière cause de variation n'est pas essentielle puisqu'on peut toujours modifier $\Omega_{1}$ de sorte que $J_{0} \Omega_{1} / \Omega$ reste constant. Nous utiliserons donc comme paramètres indépendants

$$
\frac{\omega_{1}}{\omega} \text { et } \frac{\bar{\Omega}_{1}}{\Omega}=J_{0}\left(\frac{\Omega_{1}}{\Omega}\right) \text {. }
$$

Les variations théoriques de $\sigma_{\mathrm{x}}, \sigma_{\mathrm{y}}, \sigma_{\mathrm{z}}$ sont représentées par les courbes de la figure 11. L'échelle des sensibilités est arbitraire. Les points sont expérimentaux. L'accord est relativement satisfaisant.
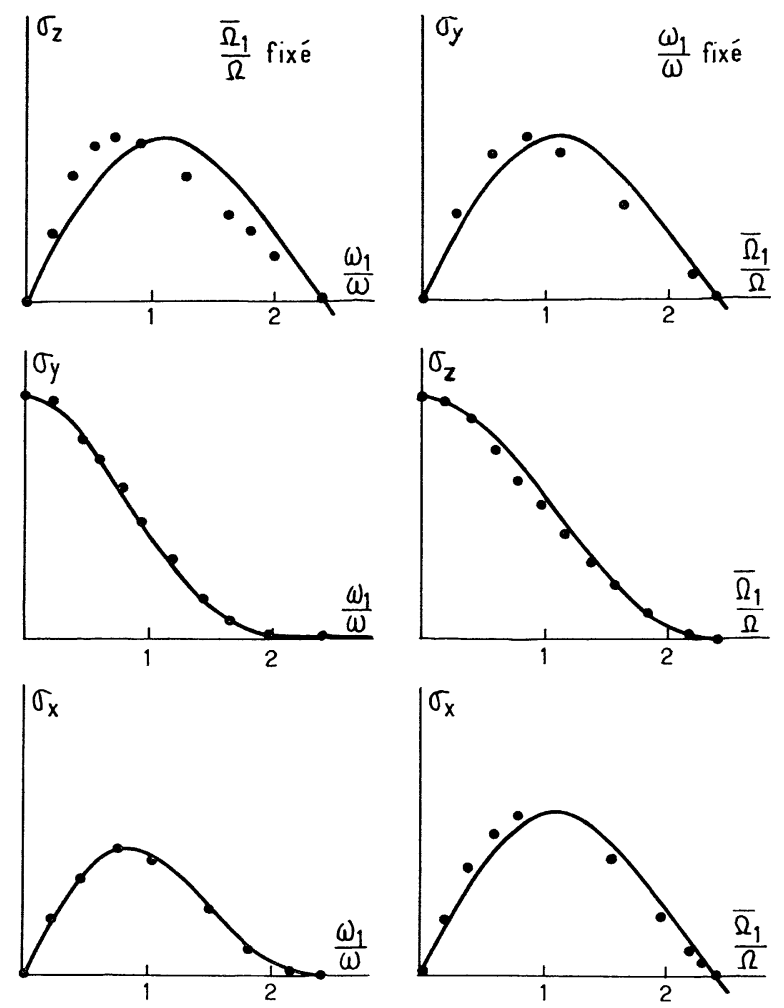

FIG. 11. - Variations, en fonction des intensités des champs de R. F., des sensibilités $\sigma_{\mathrm{x}}, \sigma_{\mathrm{y}}, \sigma_{\mathrm{z}}$ aux champs $H_{\mathrm{x}}, H_{\mathrm{y}}, H_{\mathrm{z}}$.

Les valeurs théoriques de $\omega_{1} / \omega$ et $\Omega_{1} / \Omega$ qui maximisent la sensibilité $\sigma_{\mathrm{x}}$, qui est la plus faible, sont de l'ordre de $\omega_{1} / \omega=0,84$ et $\bar{\Omega}_{1} / \Omega=1,1$. Si on prend comme référence la sensibilité maximum de la résonance paramétrique simple, les sensibilités sont alors $\sigma_{\mathrm{z}}=0,51 ; \sigma_{\mathrm{y}}=0,56 ; \sigma_{\mathrm{x}}=0,52 \Gamma / \Omega$.

Les valeurs expérimentales optimales sont en fait $\omega_{1} / \omega \approx 0,8 ; \bar{\Omega}_{1} / \Omega \approx 0,95$.

f) Nous pouvons maintenant discuter le choix de la fréquence $\Omega / 2 \pi$. Pour que $\mathscr{H}_{1} \cos \Omega t$ produise le phénomène de résonance paramétrique, il faut que $\Omega>\Gamma$, afin que la résonance en champ nul soit séparée des résonances $n= \pm 1$. Mais la sensibilité dans la direction $\mathrm{Ox}$ est proportionnelle à $\Gamma / \Omega$ (au moins au $1^{\text {er }}$ ordre en $\Gamma / \Omega$ ). La figure 12 représente la variation expérimentale en fonction de $\Omega$ de l'intensité du signal selon $O x$, prise pour la valeur optimale de $\omega_{1} / \omega$ et $\bar{\Omega}_{1} / \Omega$. On voit qu'on a intérêt à prendre $\Omega$ le plus petit possible. On est limité inférieurement par $\Omega=\Gamma$, si l'on veut que le champ $\mathfrak{H}_{1} \cos \Omega t$ continue à produire l'effet de résonance paramétrique dans la direction $\mathrm{Oy}$. On est donc amené à faire un compromis entre les deux exigences $\Omega>\Gamma$ et $\Gamma / \Omega$ maximum, ce qui impose $\Omega$ de l'ordre de quelques $\Gamma$.

On pourrait envisager une tout autre solution : $\Omega \ll \Gamma . \mathscr{H}_{1} \cos \Omega t$ produit alors un effet complètement différent de celui que nous avons étudié. Il s'agit d'un balayage lent du champ $H_{\mathrm{y}}$. On obtiendrait alors un signal modulé à $\Omega$ d'amplitude proportionnel à $\omega_{\mathrm{y}}$, 


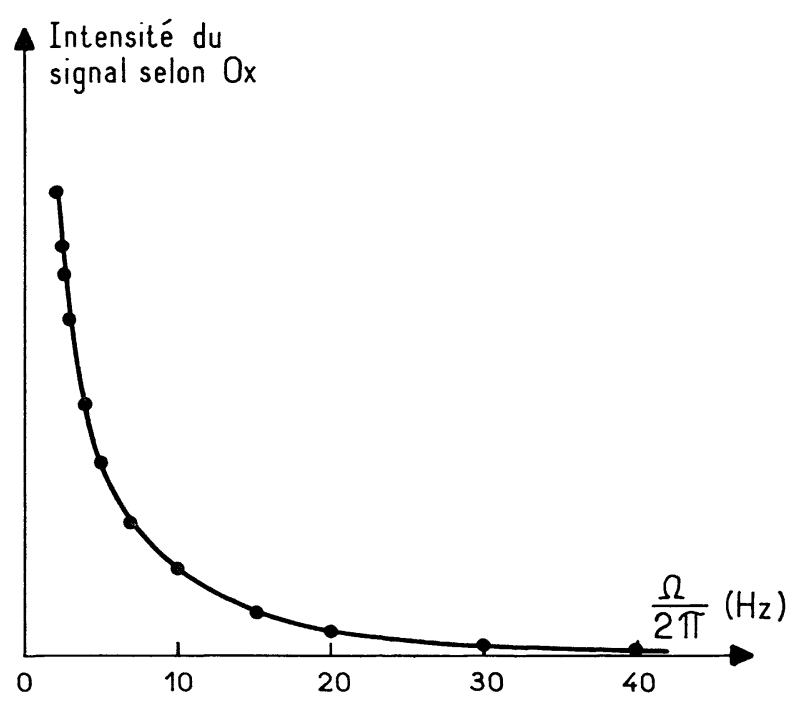

FIG. 12. - Variation, en fonction de la fréquence $\Omega / 2 \pi \mathrm{du}$ $2^{\text {e }}$ champ de R. F., de la sensibilité à $H_{\mathrm{x}}$.

et un signal de la forme $\sin \omega t \cos \Omega t$, d'amplitude proportionnelle à $\omega_{\mathbf{x}}$, avec des amplitudes facilement calculables d'après les résultats de la partie I. Toutefois, cette solution suppose une largeur de raie suffisamment importante pour qu'il existe des fréquences très petites devant $\Gamma / 2 \pi$, mais néanmoins assez grandes pour que l'amplification sélective et la détection synchrone des signaux à la fréquence $\Omega / 2 \pi$ ne nécessite pas de constantes de temps prohibitives. Les raies étant plus larges, à rapport signal sur bruit égal, la sensibilité du dispositif est alors moins grande. On peut envisager une telle solution pour des niveaux atomiques à temps de relaxation court, comme par exemple le niveau métastable $2^{3} \mathrm{~S}_{1}$ de l'hélium. Elle était exclue dans notre cas, où le temps de relaxation est de l'ordre de $1 \mathrm{~s}$.

Conclusion. - Nous avons donc mis au point deux méthodes permettant de détecter par des méthodes optiques les 3 composantes d'un champ magnétique très faible.

La première utilise les « résonances paramétriques 》 qui apparaissent en champ nul lorsqu'on balaie le champ dans une direction quelconque par rapport au champ de radiofréquence. Nous avons établi la forme exacte des signaux et nous les avons observés expérimentalement dans l'état fondamental de ${ }^{87} \mathrm{Rb}$. Nous avons montré que par des jeux de décompensation, on peut mesurer successivement chacune des composantes. Les sensibilités que l'on peut atteindre sont de l'ordre de $10^{-9} \mathrm{G}$ pour la composante du champ parallèle au champ de R. F. et 2 à $3 \times 10^{-9} \mathrm{G}$ pour les deux autres composantes. Le principal inconvénient de cette méthode est la nécessité d'une mesure successive des 3 composantes. Même si on automatise le cycle de mesure, il est relativement long (10 à $30 \mathrm{~s}$ ). Cette méthode est donc recommandée pour la mesure avec une grande précision d'un champ magnétique variant lentement.

La deuxième méthode utilise les signaux de résonance qui apparaissent en présence de deux champs de radiofréquence. Nous avons montré que ce dispositif fournit simultanément 3 signaux différents proportionnels aux 3 composantes du champ. Il se prête donc parfaitement à des mesures plus rapides, en particulier à un asservissement des 3 composantes du champ à zéro. Mais il a une sensibilité plus faible que le précédent dans un rapport de 2 à 10 suivant les composantes.

Remerciements. - Je tiens à remercier vivement C. Cohen-Tannoudji pour l'intérêt qu'il a pris à ce travail et pour ses conseils, qui ont été très utiles à l'élaboration de cet article.

Appendice. - Les deux équations différentielles [1] peuvent être remplacées par deux équations intégrales, donnant la solution en régime permanent.

$$
\begin{aligned}
M_{+}= & \exp \left(-\Gamma t+i \omega_{\mathrm{z}} t+i \frac{\omega_{1}}{\omega} \sin \omega t\right) \times \\
& \times \int_{-\infty}^{t} \exp \left(\Gamma \tau-i \omega_{\mathrm{z}} \tau-i \frac{\omega_{1}}{\omega} \sin \omega \tau\right) \\
& \times\left(\lambda-2 i \omega_{+} M_{\mathrm{z}}(\tau)\right) \mathrm{d} \tau \\
M_{\mathrm{z}}= & -i e^{-\Gamma t} \int_{-\infty}^{t} \mathrm{e}^{\Gamma \pi}\left(\omega_{-} M_{+}(\tau)-M_{-}(\tau) \omega_{+}\right) \mathrm{d} \tau
\end{aligned}
$$

On en déduit les relations de récurrence qui lient $M_{+}^{(n)}$ et $M_{z}^{(n)}$ à $M_{+}^{(n-1)}$ et $M_{z}^{(n-1)}$

$$
\begin{aligned}
M_{+}^{(n)}= & -\exp \left(-\Gamma t+i \omega_{\mathrm{z}} t+i \frac{\omega_{1}}{\omega} \sin \omega t\right) \times \\
\times & \int_{-\infty}^{t} \exp \left(\Gamma \tau-i \omega_{\mathrm{z}} \tau-i \frac{\omega_{1}}{\omega} \sin \omega_{\tau}\right) \times \\
\times & 2 i \omega_{+} M_{\mathrm{z}}^{(n-1)}(\tau) \mathrm{d}_{\tau} \\
M_{\mathrm{z}}^{(n)}= & -i e^{-\Gamma t} \int_{-\infty}^{t} \times \\
& \mathrm{e}^{\Gamma_{\tau}}\left(\omega_{-} M_{+}^{(n-1)}(\tau)-M_{-}^{(n-1)}(\tau) \omega_{+} \mathrm{z} \mathrm{d} \tau\right.
\end{aligned}
$$

avec

$$
\begin{aligned}
M_{+}^{(0)}= & \lambda \exp \left(-\Gamma t+i \omega_{\mathrm{z}} t+i \frac{\omega_{1}}{\omega} \sin \omega t\right) \times \\
& \times \int_{-\infty}^{t} \exp \left(\Gamma \tau-i \omega_{\mathrm{z}} \tau-i \frac{\omega_{1}}{\omega} \sin \omega \tau\right) \mathrm{d} \tau \\
= & \frac{\lambda J_{0}}{\Gamma-i \omega_{z}} \exp \left(i \frac{\omega_{1}}{\omega} \sin \omega t\right) \\
M_{\mathrm{z}}^{(0)}= & 0
\end{aligned}
$$

Nous avons négligé dans l'intégration de (A.5) 
tous les termes en $\Gamma / \omega, \omega_{\mathbf{z}} / \omega$. Nous les négligerons également dans la suite. De proche en proche, on trouve facilement que $M_{\mathrm{z}}^{(2 n)}=0, M_{+}^{(2 n+1)}=0$ et que $M_{+}^{(2 n)}$ est de la forme

$$
M_{+}^{(2 n)}=R^{(2 n)} \exp \left(i \frac{\omega_{1}}{\omega}\right) \sin \omega t
$$

où $R^{(2 n)}$ est relié à $R^{(2 n-2)}$ par la relation de récurrence linéaire

$R^{(2 n)}=-\frac{2 \omega_{+} J_{0}^{2}}{\Gamma\left(\Gamma-i \omega_{\mathrm{z}}\right)} \times$

$$
\times\left(\omega-R^{(2 n-2)}-\omega_{+} R^{(2 n-2) *}\right) .
$$

De plus

$$
M_{\mathrm{z}}^{(2 n+1)}=-\frac{J_{0}}{\Gamma} i\left(\omega_{-} R^{(2 n)}-\omega_{+} R^{(2 n) *}\right) .
$$

$M_{+}$et $M_{\mathrm{z}}$ s'expriment en fonction de la somme de la série $\sum_{n=0}^{\infty} R^{(2 n)}$; soit $A$ et $B$ les parties réelles et imaginaires de cette somme.

$$
\begin{aligned}
& M_{+}=(A+i B) \exp \left(i \frac{\omega_{1}}{\omega} \sin \omega t\right) \\
& M_{\mathrm{z}}=-\frac{J_{0}}{\Gamma}\left(\omega_{\mathrm{y}} A-\omega_{\mathrm{x}} B\right) .
\end{aligned}
$$

La somme de la série s'obtient facilement en sommant pour $n$ variant de 1 à $\infty$ les deux membres de (A.7). On trouve

$$
(A+i B)-R^{(0)}=-\frac{2 \omega_{+} J_{0}^{2}}{\Gamma\left(\Gamma-i \omega_{\mathrm{z}}\right)} i\left(\omega_{\mathrm{x}} B-\omega_{\mathrm{v}} A\right) .
$$

En séparant partie réelle et partie imaginaire, on obtient un système linéaire de deux équations aux 2 inconnues $A$ et $B$ dont la solution est donnée par les expressions (4) et (5).

\section{Bibliographie}

[1] Dupont-Roc (J.), Haroche (S.), Cohen-Tannoudu (C.), Phys. Letters, 1969, 28A, 638.

[2] Cohen-Tannoudji (C.), Dupont-Roc (J.), Haroche (S.), Laloe (F.), Rev. Phys. Appliquée, 1970, 5, 95 et 102

[3] Cohen-Tannoudji (C.), Dupont-Roc (J.), Haroche (S.), Laloe (F.), Phys. Rev. Lett., 1969, 22, 758.

[4] Polonsky (N.), Cohen-Tannoudji (C.), C. R. Ac. Sc., 1965, 260, 5231, J. Phys., 1965, 26, 409.

[5] Favre (C. J.), Geneux (E.), Phys. Letters, 1964, 8, 190. Alexandrov (E. B.) et Al., Sh. Eksp. Teor. Fiz, $1963,45,503$.

[6] Cohen-Tannoudji (C.), Dupont-Roc (J.), J. Physique, $1971,32$.

[7] Cette méthode de calcul par itération est analogue à celle utilisée en optique non linéaire. Voir : Blembergen (N.), Shen (Y. R.), Phys. Rev., 1964, 133, A37.
[8] Cohen-Tannoudji (C.), Haroche (S.), C. R. Ac. Sc., 1966, 262, 268.

Cohen-Tannoudji (C.), Haroche (S.), J. Phys., 1969, 30, 153.

Cohen-Tannoudji (C.), Haroche (S.), dans « Polarisation, Matière et Rayonnement ), livre de jubilé en l'honneur d'Alfred Kastler, édité par la société française de Physique, P. U. F., 1969.

[9] LANDré (C.), Thèse de $3^{\text {e }}$ cycle, Paris, 1970.

Landré (C.), COHEn-Tannoudji (C.), Dupont-Roc (J.), Haroche (S.), J. Physique, 1970, 31, 971.

[10] Lehmann (J. C.), Cohen-Tannoudji (C.), $C . R$ Ac. Sc., 1964, 258, 4463.

Pour les états excités : Hanle (W.), Z. Phys., 1924, 30, 93.

[11] Polonsky (N.), Thèse de $3^{\mathrm{e}}$ cycle, Paris, 1966. 